\title{
Mycobacterium tuberculosis: what is the role of PPE2 during infection?
}
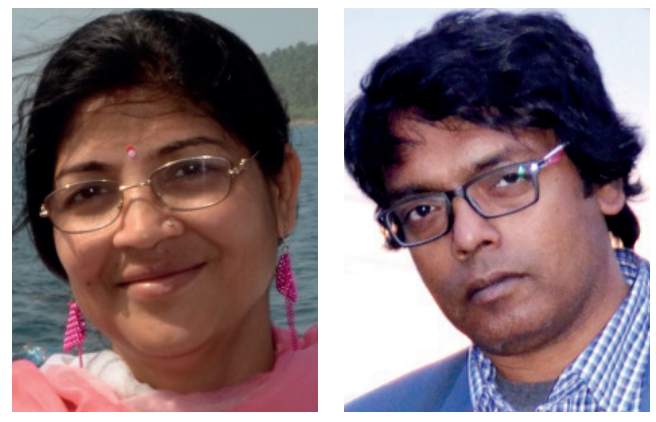

"Approximately a third of the world

population is estimated to have

latent Mycobacterium tuberculosis

infection, which is clinically

asymptomatic but possesses

significant risk of progressing to

active tuberculosis disease..."

Sangita Mukhopadhyay ${ }^{*, 1} \&$ Sudip Ghosh ${ }^{* *, 2}$

First draft submitted: 2 March 2017; Accepted for publication: 7 March 2017; Published online: 8 May 2017

Very few human pathogens possess the stealth and intellect of Mycobacterium tuberculosis $(\mathrm{Mtb})$, the bacterium responsible for TB. An extremely resilient cell wall, cunning strategies to baffle the immunologically educated host makes it a formidable pathogen. Approximately a third of the world population is estimated to have latent Mtb infection, which is clinically asymptomatic but possesses significant risk of progressing to active TB disease when the host immune system is compromised due to aging, infection with HIV among others. As the first line of host immune defense, macrophages attempt to eliminate invading pathogens initially by its innate-effector functions and later on by orchestrating the adaptive T-cell immune responses. Interestingly, macrophages also serve as the cellular niche for Mtb replication and act as reservoirs for persistent bacteria within the caseum of lung granulomas. As a master escape artist, Mtb appears to manipulate almost every arsenal of the host immune system from phagocytosis to adaptive T-cell responses and even modulate host cell metabolism to suit its intracellular lifestyle, though a complete picture is yet to emerge.

\section{Does nitric oxide play a role in human TB?}

In response to infection, one of the initial reactions of macrophages is to produce bursts of toxic reactive oxygen species and nitric oxide (NO) and its intermediates in order to kill the invading pathogens. In mice, production of $\mathrm{NO}$ is found to be one of the essential components for antimycobacterial resistance. Abrogation of inos gene, which catalyzes production of NO, could severely compromise the virulence of Mtb [1]. Mice with relatively stable and asymptomatic infection with Mtb were found to rapidly develop fatal acute illness when treated with iNOS inhibitors [2]. However, the role of $\mathrm{NO}$ in human tuberculosis is controversial. Early inhibition of mycobacterial growth by human alveolar macrophages has been

\section{KEYWORDS}

- macrophage - Mycobacterium tuberculosis • nitric oxide • PPE2 - virulence 
"In both laboratory and clinical strains, expression of ppe2 is increased when Mycobacterium tuberculosis

is exposed to the macrophage environment indicating that PPE2 may play a role in protecting the bacilli from nitric oxide and/or oxidative stress.” shown to be NO-independent. But we need to keep in mind that Mtb is primarily a human pathogen and a constant companion of anatomically modern humans during evolution and global dissemination for over last 70,000 years [3]. Then, why do they care to retain several genes like nox $R 1$, nox $R 3, \operatorname{ahp} C$ to neutralize the toxic effects of $\mathrm{NO}$ and its intermediates? Indeed, several lines of evidences suggest that NO does play a significant contributory role in human host defense against Mtb infection [4].

\section{PPE2 is upregulated during NO stress}

PPE2 belongs to a unique family of proteins called PE/PPE which represents almost $10 \%$ of the Mtb genome [5]. It was initially believed to be a potential source of antigenic variation which allows the bacterium to escape antigen-specific host responses due to the remarkable length and extensive sequence variations in some of its members [6]. Several PE/PPE proteins are now emerging as the key components of complex mycobacterial virulence mechanisms that can modulate the host cellular machinery for its survival and persistence in vivo [7]. Microarray studies have shown that expression of $p p e 2$ is upregulated in Mtb during hypoxia and $\mathrm{NO}$ stress and is also upregulated in $\operatorname{dos} S$-null mutants upon exposure to $\mathrm{NO}[8,9]$. In both laboratory and clinical strains, expression of $p$ pe 2 is increased when Mtb is exposed to the macrophage environment indicating that PPE2 may play a role in protecting the bacilli from $\mathrm{NO}$ and/or oxidative stress [10]. Nevertheless, ppe2-null mutants were found to allow higher production of nitric oxide in macrophages as compared with the wild-type strains [11].

\section{PPE2 mimics eukaryotic transcription factors}

PPE2 is probably the first Mtb protein reported to contain a functional monopartite nuclear localization signal (NLS) and a DNA-binding leucine zipper motif characteristic of many eukaryotic transcription factors [12]. Though rare in animal bacteria, several plant pathogenic bacteria possess NLS-containing effector proteins that are known to be targeted to the nucleus - for example, VirD2 and VirE2 proteins of Agrobacterium tumefaciens contain bipartite NLS which are secreted into host cells via the type IV secretion system. Nuclear targeting of effector proteins and subsequent pathology of the host cells appears to be an emerging pathogenic mechanism in bacteria.

\section{PPE2 is translocated to the nucleus to} inhibit inos transcription

Since ppe2-null mutants of Mtb allowed higher production of NO, and PPE2 contains an NLS and a DNA binding domain, it was speculated to be translocated to the nucleus to inhibit inos transcription [11,12]. PPE2 was found to utilize classical importin- $\alpha / \beta$-dependent nuclear import pathway as ivermectin blocked its nuclear import and physically interacted with importin- $\alpha$ through its NLS. Nuclear import of PPE2 was also found to be associated with decrease in the levels of inos transcripts in LPS-stimulated macrophages, indicating a direct role of PPE2 in inhibiting transcription from the inos promoter. As expected, NLS-mutants of PPE2 failed to inhibit inos transcription [12].

\section{How PPE2 gains access to the cytoplasm}

In order to be translocated to the nucleus, PPE2 first needs to gain access to the host cell cytoplasm to utilize the importin- $\alpha / \beta$ nuclear import pathway. Upon phagocytosis, Mtb is confined inside the phagaolysosomal compartment whose maturation is arrested by several Mtb proteins to prevent its destruction by lysozymes. PPE2 appears to be a secretory protein as it can be detected in the culture supernatants of a clinical strain of $M t b$ and many PPE proteins are known to be secreted through type VII ESX secretion system [11,13]. It has been reported that the phagosomes containing Mtb can allow molecules up to $70 \mathrm{kDa}$ to escape into the cytosol [14]. Nevertheless, mycobateria themselves can escape into the cytosol from phagosomes because of pore-forming activity of ESAT-6 [15].

\section{Translocated PPE2 binds to GATA-1 elements to inhibit inos transcription}

As PPE2 was also predicted to contain a leucine zipper DNA-binding motif, it was speculated that PPE2 probably binds to some crucial regulatory element of the promoter important for inos gene transcription [12]. Expression of inos is known to be predominantly regulated at the level of transcription, in addition to post-transcriptional regulation mechanisms [16]. Analyses of the inos promoter revealed presence of at least five putative GATA-1-binding sites in the promoter region and interestingly, one of the putative sequences (-16 to -25) was found to be overlapping with the TATA box close to the transcription start site. Though, the most important transcription factors required for the transcription 
of inos gene are IRF-1 and NF- $\mathrm{BB}$, evidences indicate that GATA transcription factors also play an important role in driving transcription from the promoter of the inos gene [16,17]. Since, no cognate DNA-binding sequences for PPE2 is known, it was speculated that PPE2 may inhibit inos transcription either by directly interacting with the transcription machinery or by inhibiting recruitment of crucial transcription factors by competing with their cognate regulatory binding sites. It was found that PPE2 can specifically interact with inos-specific GATA-1-binding elements but not with the cognate NF- $\kappa \mathrm{B}$ or IRF-1-binding oligonucleotides. Therefore, it appears that PPE2 possibly sterically inhibits recruitment of RNA polymerase or GATA-1 itself by occupying GATA-1-element overlapping with the TATA-box. Such transcriptional repression by competitive inhibition is widely prevalent in bacteria such as the LacI repressor protein of lac operon and SV40 T-antigen that autoregulates its expression by binding its promoter in a similar fashion $[18,19]$. Since there are four more GATA-1-binding elements present in the upstream elements [12], we presume that during infection, these sites are also occupied by PPE2 and therefore alternative mechanism of transcriptional suppression from the upstream GATA-1-bound PPE2 cannot be ruled out. Interestingly, two more $\mathrm{PE} / \mathrm{PPE}$ proteins of $\mathrm{Mtb}$, PE5 and PE15 [20] were also found to inhibit inos gene transcription and enhance bacillary survival in macrophages.

\section{Is PPE2 a virulent factor?}

Mtb lacks classical virulence factors unlike other typical bacterial pathogens - that is, toxins produced by Corynebacterium diptheri, Shigella dysenteriae or Vibrio cholerae. Therefore, in case of mycobacteria, virulence is broadly defined as factors that are important for the progression of the tuberculosis disease, usually measured in terms of mortality as well as increased bacterial load following infection. PPE2 was found to confer significant survival advantages both in vitro and in vivo to a nonpathogenic surrogate bacterium Mycobacterium smegmatis which naturally lacks this protein. Bacterial loads were found to be significantly higher in mice infected with $M$. smegmatis expressing PPE2 and correlates well with decreased levels of inos transcripts [12].

\section{Conclusion}

PPE2 appears to be secreted into the host cytoplasm during Mtb infection, translocated to the nucleus where it binds to the GATA-1 elements present in the inos promoter and sterically inhibits recruitment of the transcription machinery to inhibit transcription of inos gene. However, the exact mechanism of its interaction with the transcription machinery is yet to be understood. Since several essential gene transcriptions are also controlled by GATA-1 transcription factors, it is possible that PPE2 is selectively recruited only to those genes required to be inhibited for successful pathogenesis.

\section{Financial \& competing interests disclosure}

The Laboratories of S Mukhopadhyay and S Ghosh are supported by grants from the Department of Biotechnology (DBT), Government of India (BT/PR11605/NNTI 28/758/2014 to S Mukhopadhyay and S Ghosh; BT/PR5496/MED/29/512/2012 to S Mukhopadhyay) and the Department of Science \& Technology (EMR/ 2016/000644 to S Mukhopadhyay) and core grants from CDFD by DBT. A patent has been filed focusing PPE2 (Rv0256c) as a new drug target in tuberculosis (patent filed in USA [USPTO, 31 August 2009]). The authors have no other relevant affiliations or financial involvement with any organization or entity with a financial interest in or financial conflict with the subject matter or materials discussed in the manuscript apart from those disclosed.

No writing assistance was utilized in the production of this manuscript.

\section{References}

1 MacMicking JD, North RJ, LaCourse R, Mudgett JS, Shah SK, Nathan CF. Identification of nitric oxide synthase as a protective locus against tuberculosis. Proc. Natl Acad. Sci. USA 94(10), 5243-5248 (1997).

2 Chan J, Tanaka K, Carroll D, Flynn J, Bloom BR. Effects of nitric oxide synthase inhibitors on murine infection with
Mycobacterium tuberculosis. Infect. Immun. 63(2), 736-740 (1995)

3 Comas I, Coscolla M, Luo T et al. Out-ofAfrica migration and Neolithic coexpansion of Mycobacterium tuberculosis with modern humans. Nat. Genet. 45(10), 1176-1182 (2013).

4 Chan ED, Chan J, Schluger NW. What is the role of nitric oxide in murine and human host defense against tuberculosis? Current knowledge. Am. J. Respir. Cell. Mol. Biol. 25(5), 606-612 (2001).

5 Cole ST, Brosch R, Parkhill J et al. Deciphering the biology of Mycobacterium tuberculosis from the complete genome sequence. Nature 393(6707), 537-544 (1998).

6 Sampson SL. Mycobacterial PE/PPE proteins at the host-pathogen interface. Clin. Dev. Immunol. 2011, 497203 (2011). 
7 Ahmed A, Das A, Mukhopadhyay S. Immunoregulatory functions and expression patterns of PE/PPE family members: roles in pathogenicity and impact on anti-tuberculosis vaccine and drug design. IUBMB Life 67(6), 414-427 (2015).

8 Honaker RW, Leistikow RL, Bartek IL, Voskuil MI. Unique roles of DosT and DosS in DosR regulon induction and Mycobacterium tuberculosis dormancy. Infect. Immun. 77(8), 3258-3263 (2009).

9 Voskuil MI, Schnappinger D, Visconti KC et al. Inhibition of respiration by nitric oxide induces a Mycobacterium tuberculosis dormancy program. J. Exp. Med. 198(5), 705-713 (2003)

10 Homolka S, Niemann S, Russell DG, Rohde $\mathrm{KH}$. Functional genetic diversity among Mycobacterium tuberculosis complex clinical isolates: delineation of conserved core and lineage-specific transcriptomes during intracellular survival. PLoS Pathog. 6(7), e1000988 (2010)

11 Bhat KH, Das A, Srikantam A, Mukhopadhyay S. PPE2 protein of
Mycobacterium tuberculosis may inhibit nitric oxide in activated macrophages. Ann. NY Acad. Sci. 1283, 97-101 (2013).

12 Bhat KH, Srivastava S, Kotturu SK, Ghosh S, Mukhopadhyay S. The PPE2 protein of Mycobacterium tuberculosis translocates to host nucleus and inhibits nitric oxide production. Sci. Rep. 7, 39706 (2017).

13 Abdallah AM, Verboom T, Weerdenburg EM et al. PPE and PE_PGRS proteins of Mycobacterium marinum are transported via the type VII secretion system ESX-5. Mol. Microbiol. 73(3), 329-340 (2009).

14 Teitelbaum R, Cammer M, Maitland ML, Freitag NE, Condeelis J, Bloom BR. Mycobacterial infection of macrophages results in membrane-permeable phagosomes. Proc. Natl Acad. Sci. USA 96(26), 15190-15195 (1999).

15 Smith J, Manoranjan J, Pan M et al. Evidence for pore formation in host cell membranes by ESX-1-secreted ESAT- 6 and its role in Mycobacterium marinum escape from the vacuole. Infect. Immun. 76(12), 5478-5487 (2008).
16 Pautz A, Art J, Hahn S, Nowag S, Voss C, Kleinert H. Regulation of the expression of inducible nitric oxide synthase. Nitric Oxide 23(2), 75-93 (2010).

17 Deng WG, Wu KK. Regulation of inducible nitric oxide synthase expression by p 300 and p50 acetylation. J. Immunol. 171(12), 6581-6588 (2003).

18 Chen B, de Crombrugghe B, Anderson WB, Gottesman ME, Pastan I, Perlman RL. On the mechanism of action of lac repressor. Nat. New Biol. 233(37), 67-70 (1971).

19 Rio DC, Tjian R. SV40 T antigen binding site mutations that affect autoregulation. Cell 32(4), 1227-1240 (1983).

20 Tiwari BM, Kannan N, Vemu L, Raghunand TR. The Mycobacterium tuberculosis PE proteins Rv0285 and Rv1386 modulate innate immunity and mediate bacillary survival in macrophages. PLoS ONE 7(12), e51686 (2012). 